\title{
Ageing and maximal physical performance
}

\author{
Harri Suominen
}

Received: 26 August 2010 / Accepted: 20 October 2010 /Published online: 10 November 2010

(C) European Group for Research into Elderly and Physical Activity (EGREPA) 2010

\begin{abstract}
Elite master athletes with long-term devotion to physical training offer an economical means of investigating the effects of ageing and habitual exercise on maximal physical performance. Ideally, individuals with optimised living habits throughout their lives could serve as a human model of 'primary' or 'inherent' ageing, where age-related changes are not confounded by sedentary life-style and associated chronic diseases. A limitation of this approach is the selection bias as those with inherently high physical capacity and good health participate in competitive sports. The age-related decrements remain obvious, but the record performances of master athletes competing in running and jumping events are preserved at an extraordinary high level until old age. Similarly, underlying capacities such as muscle strength, power and endurance remain far above the age norms, thus providing superior functional reserves for activities of daily living. Nevertheless, even the best records may overestimate the age decrements. It is obvious that the older champions have never trained and performed as well as the current younger athletes, and that the performance of individual athletes retaining or increasing their training volume and intensity show smaller decrements over the years. The plasticity of individual development is preserved in later life thus making it possible, at least for some time, to modify the age-associated decline in the different aspects of maximal physical performance.
\end{abstract}

Keywords Ageing · Master athletes · Sport performance Functional capacity

\footnotetext{
H. Suominen $(\bowtie)$

Department of Health Sciences, University of Jyväskylä,

P.O. Box 35 (LL), 40014 Jyväskylä, Finland

e-mail: harri.k.suominen@jyu.fi
}

\section{Introduction}

Preserving adequate physical performance is an essential element of a healthy and productive life among the ageing population. The greater the maximal performance in physiological components such as muscle strength, power and endurance, the greater is the reserve capacity for the physical performance of activities of daily living and the potential for the continuation of independent life in older adults. A concrete example is the importance of maintaining adequate mobility when attempting to successfully negotiate crossing a busy street. Young and fit subjects can easily cope with the demands of the traffic light-controlled pedestrian clearance period (by running if necessary), while this will be much more difficult for the average 80 -year-old man or woman whose maximal walking speed is close to the critical level of the $1.2 \mathrm{~m} / \mathrm{s}$ required to beat the traffic.

Age-associated deterioration in performance can be attributed to both primary aging processes and to life-style factors such as declines in the amount and intensity of physical activity. However, the changes in physical activity among the ageing population are not unequivocal. While older populations are generally less active than young adults, the cohort studies in countries such as Finland have shown that the frequency and intensity of physical activity have increased in older adults during the last 20 years [1]. There is also a possibility of a polarisation phenomenon (see Hirvensalo and Lintunen, in this issue). At the same time as the proportion of inactive people is increasing, so too are the numbers of active individuals in different age groups.

Master athletes are active exercisers and competitors who participate in international, national or local competitions for 'masters', 'veterans' or 'seniors' [2-4]. In general, 
such competitions are divided into 5-year categories, with the minimum age typically being 35 or even lower, thus providing a continuum from youth and adult sports to training and competitions in old age. Currently, most outdoor and indoor sports have their own masters' competitions attracting thousands of competitors in international events such as track and field.

For exercise gerontologists, master athletes provide an interesting population to study. Although elite athletesfemales in particular-continue to represent a small proportion of their cohort, individuals with long-term devotion to physical training offer an economical means of investigating the role of exercise in the age-related decrements in physiological capacities and function. Highly motivated athletes provide official and controlled physical performance data, and they offer a barometer of what is possible in physical ageing $[2,5]$. Ideally, master athletes with optimised living habits throughout their lives could provide a human research model of 'primary' or 'inherent' ageing, where age-related physiological changes are less influenced by confounding factors such as sedentary lifestyle and associated chronic diseases [4, 6-11].

This article is a brief overview of the age-associated changes in sport performance in master athletes. Reviewing the current status and transitions in record performances in different age groups together with examples of individual development and underlying trajectories of physical training and functional capacity provides a lifecourse perspective on the changes in maximal physical performance. Special attention is paid to track-and-field athletes competing in running and jumping events, where muscle strength and power or endurance are essential elements of performance.

\section{Record performances}

In organised masters' sports such as track and field, the official record performances offer a well-documented, controlled database for describing maximal physical performance throughout the life span and comparing the age-related changes in athletic events imposing different demands on training and functional abilities. Accordingly, an increasing number of articles and book chapters have highlighted the value of athletics records in providing an insight into ageing and performance (for recent reviews see $[2,4,12])$. Taking the absolute best records in each age category provides a straightforward approach to the upper limits of human performance compared to studies calculating averages from different sources of statistics compiled for athletes or trying to obtain representative performance results for all athletes participating in given sports.
Sprint running

A good example of a track athletic event requiring strength and power is the $100-\mathrm{m}$ sprint, where great many highly trained athletes regularly compete at a high international level. Adequate reference data for maximal running speed in untrained men and women across the life span are lacking, but the superior performance of trained sprinters is obvious. The current world records in the $100-\mathrm{m}$ sprint (Fig. 1) [13] indicate that there is a modest curvilinear decline in running speed until approximately 80 years of age in men (about $0.6 \%$ per year) and 75 years of age in women $(0.7 \%$ per year), whereupon the exponential decline, which is typical for most physical performances, becomes more evident.

Critical factors behind the fact that even elite sprinters show a distinct decline in maximal running speed seem to be decreased stride length, reduced ability to produce efficient ground reaction forces and decreased lower extremity stiffness to tolerate the higher impact forces involved in the fast transition from the braking to push-off phases $[11,14,15]$. This may, in part, be associated with the reduced volume and intensity of strength training and consequent loss of muscle mass in the older age groups [4]. When elderly sprinters participated in a periodised strength training program, in which heavy-resistance exercises were combined with explosive types of weight training and plyometric exercises, their explosive force production, power characteristics and the cross-sectional area of type IIa fibres significantly improved [16].

However, it is obvious that the older champions represent a cohort that has never performed as well as their present-day young counterparts [10]. In fact, longitudinal

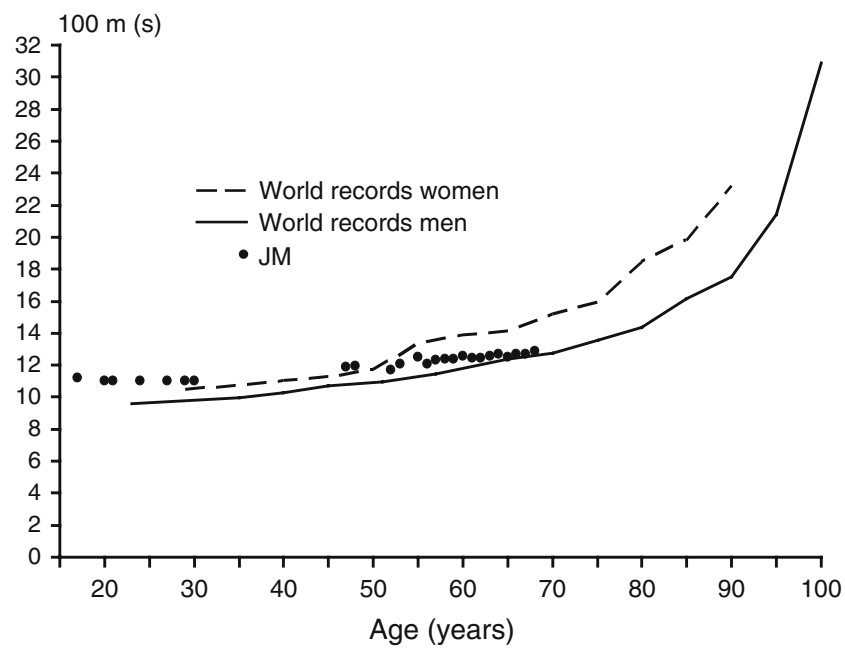

Fig. 1 Official world records for the 100-m track event in different age categories for men and women [13]. The figure also shows individual longitudinal data for a male Finnish sprinter (JM). Adapted from [4] 
data such as the individual-best $100-\mathrm{m}$ performance times of JM, the Finnish men's record holder in the age categories of 55-59, 60-64 and 65-69 years and world champion in 2007 and 2009, show a much smaller decrement in running speed over the years $(0.3 \%$ per year until age 68$)$ compared to the decline estimated from the world records. As more elite competitors continue to train and participate in the masters' athletics in the older age groups, it is likely that the current records, even in this highly competed event, will further improve.

The improvement in record performances in the older age groups over the last 25 years can be seen in a Finnish database for the best times in the 400-m track race [17]. While it has been difficult for younger adults to better the previous records, the increased number of active master competitors has clearly improved the best times in the oldest men and women [4].

Compared to the $100-\mathrm{m}$ sprint, the age-related decline in running speed estimated from the world records [13] remains somewhat greater $(0.8 \%$ per year until age 80 in men and $1.0 \%$ per year until age 75 in women) in this type of race where, in addition to strength and power, the capacity for anaerobic glycolytic metabolism becomes increasingly important $[12,18]$. However, trained athletes continue to maintain superior levels of performance throughout their lives. The world's best 70 -year-old male sprinter has run $400 \mathrm{~m}$ in $59 \mathrm{~s}$ and the world's best 80 -yearold sprinter the same distance in $70 \mathrm{~s}$, while about $20 \%$ of average men at $65-74$ and $40 \%$ at $75-84$ report that they cannot even walk $500 \mathrm{~m}$ without difficulty [19].

\section{Jumping}

Athletic events such as the high jump provide somewhat different curves (Fig. 2). Although the world records [13] are again excellent in comparison with what can be presumed to be the performance levels in the general population, the decline in performance is steeper already in middle age $(0.9 \%$ per year) and more linear throughout the age range than that shown for running speed in the previous examples. This may, in part, be due to the more complex mixture of strength, power, flexibility and technical skill needed in the high jump than in events such as sprint running. Differences in competitive status, training volume and intensity, and the use of different jumping technique by the younger compared to older athletes are also likely to play a role [4]. In the absence of any major changes in training or untoward injuries, longitudinal data should thus indicate a smaller age-related decline. This is supported by the results of athlete HS in the figure, who has retained a similar jumping technique and training volume when competing in the high jump. In the case of moderate training status and level of performance in adulthood, the

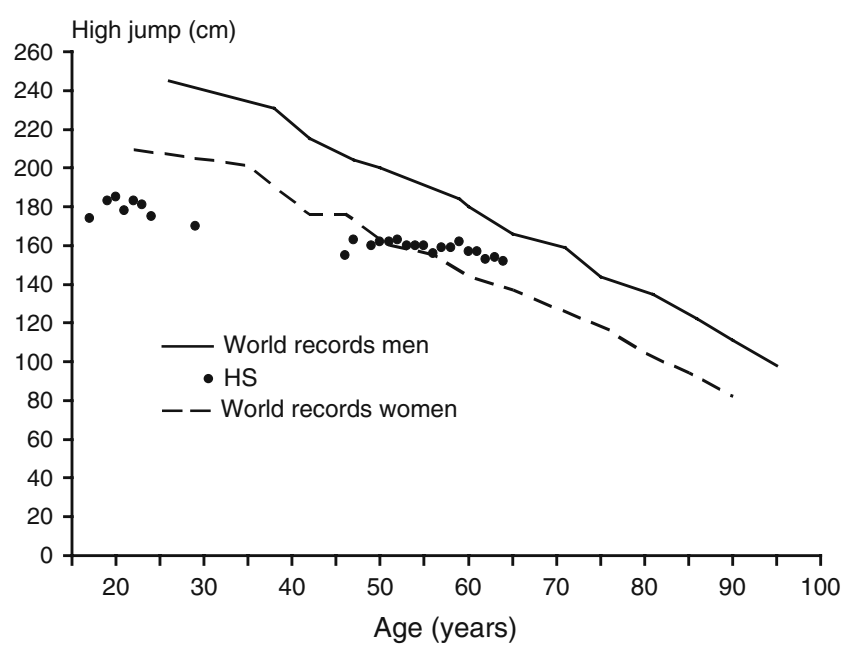

Fig. 2 Official world records for high jump in different age categories for men and women [13]. The figure also shows individual longitudinal data for a Finnish jumper (HS). Adapted from [4]

plasticity of individual development makes it possible, at least for some time, to postpone the age-related decline or even to improve performance (cf. Conzelmann [20] and Fig. 4).

\section{Long-distance running}

Long-distance running such as the marathon, which is an extreme sport on account of its high demands on aerobic power and muscle endurance, also shows excellent levels of performance in older athletes competing continuously in that event (Fig. 3) [13, 21]. The open age world record for men has been achieved at age 35 , and official results have been recorded until the 10th decade in both men and women. As with shorter running distances, the record performances do not dramatically decrease until 75 years of

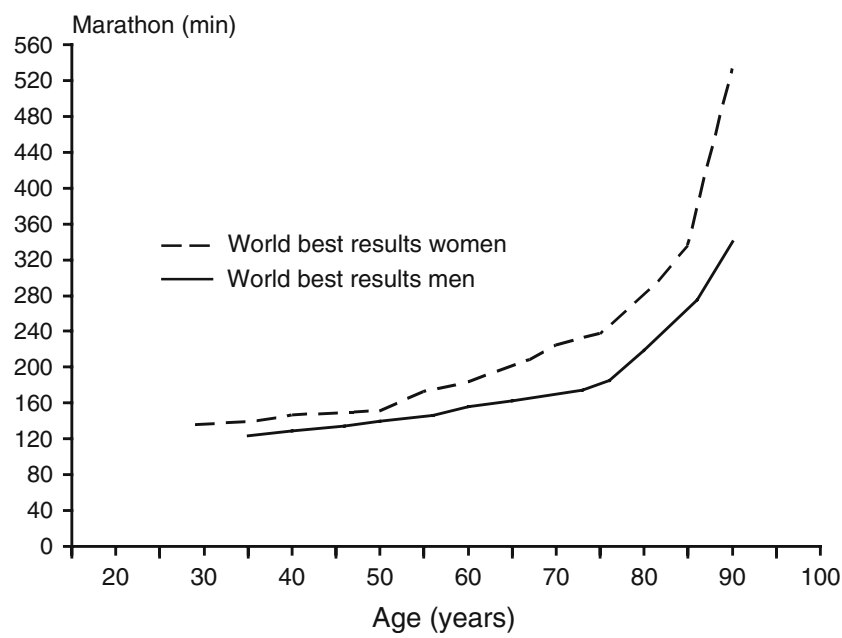

Fig. 3 World all-time best results for marathon running in different age categories for men. Data from [13] and [21] 
age (less than $1 \%$ per year in both men and women in terms of running speed). For comparison, the best 75 -year-old marathon runner would be able to cover a distance of above $2.7 \mathrm{~km}$ in 15 continuous Cooper tests (12-min run), while the average test results for young male populations remain below $2.5 \mathrm{~km} \mathrm{[22].}$

Among the physiological determinants of endurance exercise performance, a progressive reduction in maximal oxygen uptake, with contributing factors such as decreased maximal stroke volume, heart rate and arterio-venous $\mathrm{O}_{2}$ difference, appears to be the primary mechanism associated with the decline in performance with age $[3,23]$. Although the slope of the age-related decline in aerobic capacity in endurance-trained athletes resembles that in untrained persons [23, 24], this decline cannot be solely attributed to ageing as these athletes also reduce their training intensity and volume as they get older [3, 23]. On the other hand, the age-related decline in aerobic capacity in controls may be biased in that the subjects tested in the oldest age groups probably represent individuals with better health and fitness than the average sedentary population [4]. It is also noteworthy that, where the slopes of the decline are similar, the relative difference in aerobic capacity between endurance athletes and non-athletes is actually greater with ageing.

\section{Plasticity of performance}

As suggested above, the volume and intensity of physical training and the plasticity of performance affect both athletic ability and the age-associated decline in performance. Figure 4 shows the individual development of performance in an athlete with relatively stable and moderate training volume but with distinct changes in the type of training engaged in over the years. The athlete competed in power events such as hurdling, high jump and decathlon until age 25, when he turned to aerobic events such as endurance running and cross-country skiing. At age 40, he started competing in masters' track and field and changed his training focus towards more anaerobic events such as long hurdles. Since age 47 , he has trained mainly muscle strength and power and competed in the same events as he did at around age 20 .

The changes in training modalities of this athlete have clearly influenced the development of his record performances and measures of muscle strength and aerobic capacity. At the same time as his maximal oxygen uptake increased with aerobic training, his muscle strength decreased. His aerobic capacity then declined during the 'anaerobic' training period while his $400-\mathrm{m}$ race record was better maintained. Increasing the amount of strength and power training rapidly improved his performance in the

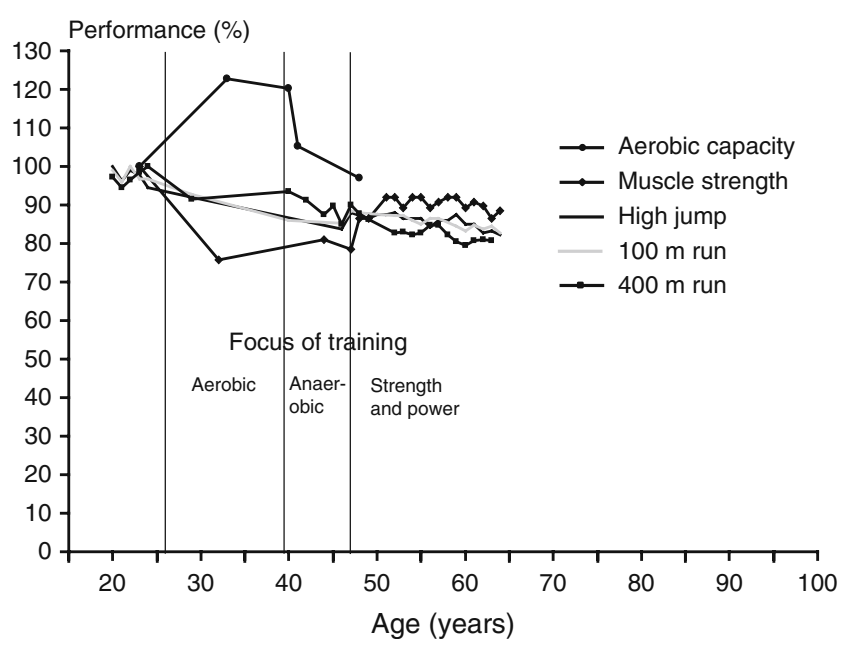

Fig. 4 Individual development of performance in different running and jumping events together with changes in aerobic capacity and muscle strength over the years in a track-and-field athlete with regard to the focus of training. The results are percentages of performance at age 20-25. The track records represent official competition results, aerobic capacity a direct measurement of maximal oxygen uptake and muscle strength one repetition maximum in bench press (Suominen, unpublished observations)

bench press as well as in the high jump and 100-m sprint, and maintained his level of performance in these events virtually unchanged over the following 15 years. On the other hand, his $400-\mathrm{m}$ race records seemed to deteriorate due the loss of longer sprints in training.

Genetic influences play an important role in explaining individual differences in exercise participation [25] and training responses [26] in general populations (see Kujala, in this issue). Although scientific evidence in master athletes is lacking, good inheritance of physical traits as well as optimal exercise habits and acquisition of adequate sport skills from childhood through adolescence are likely to be fundamental to achieving excellent athletic abilities in adulthood and ageing. The present example shows that later-life transitions in the types of physical training can also considerably modify the age-associated changes in the different aspects of maximal physical performance. If more elite athletes were to utilise their genetic potential for training and competing in the older age groups, they could further raise the upper limits of human performance and reduce the overestimation of age decrements in the oldest groups, particularly in women.

It is important to note that health complications may occur during sports and that the occurrence of severe injuries and diseases usually ends the athletic career, thus further selecting the athletes competing in old age. However, the risk for most chronic diseases is reduced among master athletes, and there seems to be no such health-related reasons as to why those who have good training background and feel healthy should not participate in master athletics $[27,28]$. 


\section{Concluding remarks}

Elite master athletes with long-term devotion to physical training are challenging present estimates of age-related changes in maximal physical performance. Although a distinct age decrement remains, the official records of athletes competing in track and field events such as running and jumping show that sport performance can be preserved at an extraordinary high level well into old age. Similarly, there is emerging evidence that underlying factors such as muscle strength, power and endurance are maintained far above the age norms, thus providing superior functional reserves for activities of daily living.

The absolute best records in each age category offer a straightforward approach to the upper limits of performance, but nevertheless continue to overestimate the age decrements in physical function. It is obvious that the older champions have never trained and performed as well as their present-day younger counterparts, and that the individual athletes retaining or increasing their training volume and intensity show smaller decrement over the years. The plasticity of individual development is preserved in later life thus making it possible, at least for some time, to modify the age-associated decline in the different aspects of maximal physical performance.

Although the intensive physical training practised by athletes is beyond the scope of most sedentary older populations, there is a lesson to be learned from the fortunate individuals with good physical inheritance, health habits and motivation throughout the life course. As stated by Spirduso et al. [2], master athletes raise both physical and psychological ceilings and shatter the barriers of expectations that society has for the elderly.

\section{References}

1. Hirvensalo M, Mäkilä P, Parkatti T, Kannas S, Huovinen P, Oinonen M-L, Lampinen P, Äijö M (2006) Physical exercise in 1988, 1996 and 2004 among 65-69-year-old residents of Jyväskylä. In: Heikkinen E, Kauppinen M, Salo P, Suutama T (eds) Are the health and functional capacity of people aged 65-69 improving and their leisure activity increasing? Observations from cohort comparisons conducted as part of the Evergreen project in 1988, 1996 and 2004. Studies in Social Security and Health 83: 133-150

2. Spirduso WW, Francis KL, MacRae PG (2005) Physical dimensions of aging. Human Kinetics, Champaign

3. Reaburn P, Dascombe B (2008) Endurance performance in masters athletes. Eur Rev Aging Phys Act 5:31-42

4. Suominen H, Korhonen MT (2010) Sport performance in master athletes: age-associated changes and underlying neuromuscular factors. In: Komi PV (ed) Neuromuscular aspects of sport performance. Volume XVIII of the Encyclopedia of Sports Medicine. An IOC Medical Commission Publication. Wiley-Blackwell, Oxford, pp 270-282

5. Harridge SD, Suominen H (2003) Physical activity in the elderly. In: Kjaer M, Krogsgaard M, Magnusson P, Engebretsen L, Roos H, Takala T, Woo SL-Y (eds) Textbook of sports medicine. Basic science and clinical aspects of sports injury and physical activity. Blackwell Science, Oxford, pp 337-354

6. Maharam LG, Bauman PA, Kalman D, Skolnik H, Perle SM (1999) Masters athletes. Factors affecting performance. Sports Med 28:273-285

7. Hawkins SA, Wiswell RA, Marcell TJ (2003) Exercise and the master athlete - a model of successful aging? J Gerontol A Biol Sci Med Sci 58:1009-1011

8. Tanaka H, Seals DR (2003) Invited review: dynamic exercise performance in masters athletes: insight into the effects of primary human aging on physiological functional capacity. J Appl Physiol 95:2152-2162

9. Rittweger J, Kwiet A, Felsenburg D (2004) Physical performance in elite athletes - challenging the limits of physiology. J Musculoskel Neuron Interact 4:159-160

10. Lazarus NR, Harridge SD (2007) Inherent ageing in humans: the case for studying master athletes. Scand J Med Sci Sports 17:461463

11. Korhonen MT (2009) Effects of aging and training on sprint performance, muscle structure and contractile function in athletes. Studies in Sport, Physical Education and Health 137, Ph.D. thesis, University of Jyväskylä

12. Reaburn P, Dascombe B (2009) Anaerobic performance in master athletes. Eur Rev Aging Phys Act 6:39-53

13. World Masters Athletics (2010). Available at http://www.worldmasters-athletics.org/records. Accessed 18 August 2010

14. Korhonen MT, Mero A, Suominen H (2003) Age-related differences in 100-m sprint performance in male and female master runners. Med Sci Sports Exerc 35:1419-1428

15. Korhonen MT, Mero A, Alen M, Sipilä S, Häkkinen K, Liikavainio T, Viitasalo JT, Haverinen MT, Suominen H (2009) Biomechanical and skeletal muscle determinants of maximum running speed with aging. Med Sci Sports Exerc 41:844-856

16. Cristea A, Korhonen MT, Häkkinen K, Mero A, Alen M, Sipilä S, Viitasalo JT, Koljonen MJ, Suominen H, Larsson L (2008) Effects of combined strength and sprint training on regulation of muscle contraction at the whole-muscle and single-fibre levels in elite master sprinters. Acta Physiol (Oxford) 193:275-289

17. Dunkel G (2010). Available at http://www.kolumbus.fi/geodun/ 400m.htm. Accessed 18 August 2010

18. Korhonen MT, Suominen H, Mero A (2005) Age and gender differences in blood lactate response to sprint running in elite master athletes. Can J Appl Physiol 30:647-665

19. Aromaa S, Koskinen S (eds) (2004) Health and functional capacity in Finland. Baseline results of Health 2000 health examination survey. Publications of the National Public Health Institute B12, Helsinki

20. Conzelmann A (1997) The plasticity of courses of physical performance in the second part of life. In: Huber G (ed) Healthy aging, activity, and sports (Proceedings, PAAS IV). Health Promotion, Hamburg, pp 435-445

21. Gasselsberger M (2010). Available at http://www.mastersathletics. net. Accessed 18 August 2010

22. Santtila $M$, Kyröläinen $H$, Vasankari $T$, Tiainen $S$, Palvalin $K$, Häkkinen A, Häkkinen K (2006) Physical fitness profiles in young Finnish men during the years 1975-2004. Med Sci Sports Exerc 38:1990-1994

23. Tanaka H, Seals DR (2008) Endurance exercise performance in masters athletes: age-associated changes and underlying physiological mechanisms. J Physiol 586:55-63

24. Harridge SD, Magnusson G, Saltin B (1997) Life-long endurancetrained elderly men have high aerobic power, but have similar muscle strength to non-active elderly men. Aging (Milano) 9:80 87

25. Stubbe JH, Boomsma DI, Vink JM, Cornes BK, Martin NG, Skytthe A, Kyvik KO, Rose RJ, Kujala UM, Kaprio J, Harris JR, 
Pedersen NL, Hunkin J, Spector TD, de Geus EJ (2006) Genetic influences on exercise participation in 37,051 twin pairs from seven countries. PLoS One 1:e22

26. Bouchard C, Rankinen $T$ (2001) Individual differences in response to regular physical activity. Med Sci Sports Exerc 33: S446-S451
27. Kujala UM, Sarna S, Kaprio J, Koskenvuo M, Karjalainen J (1999) Heart attacks and lower-limb function in master endurance athletes. Med Sci Sports Exerc 31:1041-1046

28. Kettunen JA, Kujala UM, Kaprio J, Sarna S (2006) Health of master track and field athletes: a 16-year follow-up study. Clin J Sport Med 16:142-148 